\title{
High-Intensity Focused Ultrasound: A Satisfactory, Non-invasive Procedure for Crow's Feet Wrinkles
}

\author{
Gyu Sik Jung ${ }^{1}$ \\ In Kook Cho ${ }^{2}$ \\ Hyung Min Sung ${ }^{3}$
}

${ }^{1}$ GLAD Plastic Surgery Clinic, Daegu, Korea
${ }^{2}$ Lavian Plastic Surgery Clinic, Seoul, Korea
${ }^{3}$ V Plastic Surgery Clinic, Daegu, Korea

Received August 4, 2019

Accepted September 5, 2019

\section{Correspondence}

Gyu Sik Jung

GLAD Plastic Surgery Clinic, 31 Dongsung-ro

5-gil, Jung-gu, Daegu 41941, Korea

Tel.: +82-53-710-0103

Fax: +82-53-710-0133

E-mail: jksheartanaver.com

(C) Korean Society for Laser Medicine and Surgery

(c) This is an open access article distributed under the terms of the Creative Commons Attribution NonCommercial License (http://creativecommons.org/ licenses/by-nc/4.0) which permits unrestricted noncommercial use, distribution, and reproduction in any medium, provided the original work is properly cited.

\begin{abstract}
Background and Objectives
High-intensity focused ultrasound (HIFU) has been developed as an effective, non-invasive, skin-tightening method in response to the increasing demand for improvements in skin laxity and tightening with minimal risk and recovery time. This study evaluated the efficacy and safety of HIFU for non-invasive skin tightening of crow's feet wrinkles, with the aim of determining how long the tightening can be maintained.
\end{abstract}

\section{Materials and Methods \\ Between January and March 2019, 21 female patients with crow's feet wrinkles were treated with HIFU. The treatment involved 200 shots, three times every 2 weeks. Three blinded, experienced plastic surgeons and patients evaluated satisfaction at 2 weeks after the first procedure, 2 weeks after the second procedure, 2 weeks after the third procedure, and 6 weeks after the first procedure based on photographs according to the Global Aesthetic Improvement Scale (GAIS). The Friedman test was used to compare data.}

\section{Results}

Of the 21 patients treated using HIFU, one was lost to follow-up for nonstudy-related reasons. Therefore, 20 patients were evaluated and ranged in age from 28 to 48 years. Plastic surgeons' GAIS scores were 2.6, 2.3, 1.7, and 1.3 and patients' GAIS scores were 2.6, 2.2, 1.8, and 1.4 at 2 weeks after the first procedure, 2 weeks after the second procedure, 2 weeks after the third procedure, and 6 weeks after the third procedure. No serious adverse effects were observed.

\section{Conclusion}

The aging face with crow's feet wrinkles can be improved by using HIFU, while minimizing epidermal and dermal injury.

\section{Key words}

Skin aging; High-intensity focused ultrasound therapy; Skin wrinkling 


\section{INTRODUCTION}

Crow's feet wrinkles are characterized as laugh lines around the lateral aspect of the eyes. Static fine wrinkles around the eyes and dynamic wrinkles caused by movement of the orbicularis oculi muscle develop with aging. Non-invasive skin tightening is superior to invasive or surgical skin tightening in terms of rapid return to work, short recovery time, and low risk of adverse events. Because of these advantages, patients who desire a skintightening procedure prefer non-invasive skin tightening to invasive or surgical skin tightening."

To meet patients' demand for non-invasive skin tightening, numerous devices besides the popular botulinum toxin procedure have been developed. Specifically, laser and radiofrequency devices have been developed to resolve skin wrinkling. Botulinum toxin treatment has a disadvantage in that it causes an awkward expression by reducing movement of the eyes. Recently, high-intensity focused ultrasound (HIFU) was developed as an effective non-invasive skin-tightening method. HIFU is able to heat tissue to greater than $60^{\circ} \mathrm{C}$ and produce a small thermal coagulation zone to reach the mid- to deep reticular layers of the dermis and subdermis while minimizing overlying papillary dermal and epidermal injury. The delivery of HIFU to a targeted zone in the superficial musculoaponeurotic system (SMAS) provokes the immediate contracture of denatured collagen, and initiation of neocollagenesis and collagen remodeling. This action of HIFU provokes non-invasive skin tightening and lifting of sagging facial skin.

However, certain factors including a lack of efficacy, persistence, and reliability have limited its replacement of invasive surgical procedures. ${ }^{2,3}$ The purposes of this study were to evaluate the efficacy and safety of HIFU for crow's feet wrinkles, and to determine how long the tightening of crow's feet wrinkles can be maintained.

\section{MATERIALS AND METHODS}

Between January and March 2019, 21 patients with crow's feet wrinkles were treated with HIFU (I-SHURINK ${ }^{\circledR}$; Classys Inc., Seoul, Korea; Fig. 1) using 4-MHz, 2-mm depth transducers (I-SHURINK MF2). Treatment was performed by the same surgeon and involved 200 shots, three times every 2 weeks. Informed consent was obtained from all patients, and the study was performed according to the Helsinki Declaration.

The exclusion criteria were cervicofacial, neurologic, or vascular facial disease; pregnancy or breastfeeding; lo- cal skin diseases that might alter wound healing; history of psychiatric illness, soft tissue augmentation material, cardiopathy, diabetes, facial or neck skin conditions, facial surgery; receipt of an antiaging procedure in the preceding 6 months; and active systemic or local infections.

\section{Procedure}

Ten percent lidocaine, as a topical anesthetic ointment (EMLA, AstraZeneca, Sdertlje, Sweden), was applied to the periocular area for 30 minutes before the procedure. The ointment was washed off with mild soap and water immediately before the procedure. Then ultrasound gel was applied to the periocular area, and the transducer was placed firmly on the targeted skin surface and pressed uniformly to ensure complete contact with the skin. Treatment exposure was initiated (2-mm depth transducers; $0.4 \mathrm{~J} / \mathrm{mm}^{2}$ ), with a line of individual ultrasound pulses being delivered within approximately 2 seconds. Then, the transducer was slid to the next location and repositioned 2-mm laterally such that it was adjacent and parallel to the previous treatment line. Complete treatment of the face required 10 to 15 minutes. The ultrasound gel was washed off. Patients experienced mild redness and swelling that could persist for several days.

\section{Measurement}

We compared the preoperative and postoperative measurements with the Global Aesthetic Improvement Scale (GAIS) at 2 weeks after the first procedure, 2 weeks after the second procedure, 2 weeks after the third procedure, and 6 weeks after the third procedure. Each scoring sheet

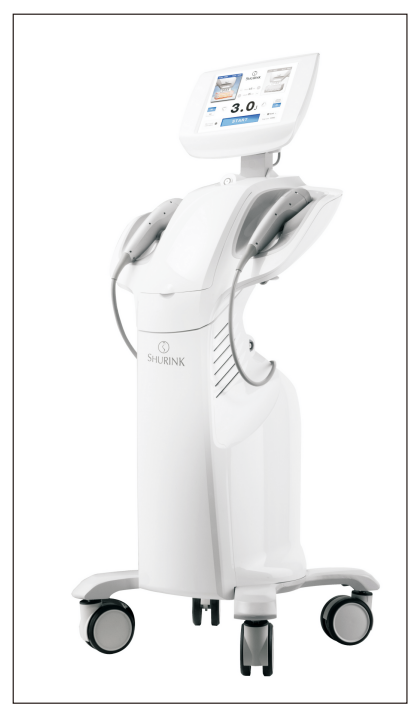

Fig. 1. High-intensity focused ultrasound (I-SHURINK ${ }^{\circledR}$ ). 
was independently assessed by 3 blinded, experienced evaluators ( 3 plastic surgeons), and the plastic surgeons and patients' scores were compared.

\section{Statistical analysis}

The Friedman test was used to compare the scores of patients at pretreatment, and at 2 and 4 months after treatment. A $p$-value $<0.05$ was considered statistically significant. Statistical analyses were performed using SPSS, version 20.0 (IBM Corp., Armonk, NY, USA).

\section{RESULTS}

All patients were treated using HIFU, and 1 patient was lost to follow-up for non-study-related reasons. Therefore, in our study, 20 female patients were evaluated and ranged in age from 28 to 48 years. There was no case of edema or erythema, linear striations, hypopigmentation, hyperpigmentation, ulceration, and erosion. There were also no adverse events, such as nerve or muscle dysfunction, severe pain, bruising, and bleeding.

Plastic surgeons' GAIS scores were 2.6, 2.3, 1.7, and 1.3 and patients' GAIS scores were 2.6, 2.2, 1.8, and 1.4

Table 1. Global Aesthetic Improvement Scale (GAIS)

\begin{tabular}{lcccc}
\hline & $\begin{array}{c}2 \text { weeks after } \\
\text { first procedure }\end{array}$ & $\begin{array}{c}2 \text { weeks after } \\
\text { second procedure }\end{array}$ & $\begin{array}{c}2 \text { weeks after } \\
\text { third procedure }\end{array}$ & $\begin{array}{c}6 \text { weeks after } \\
\text { first procedure }\end{array}$ \\
\hline Plastic surgeons' GAIS scores & 2.6 & 2.3 & 1.7 & 1.3 \\
Patients' GAIS scores & 2.6 & 2.2 & 1.8 & 1.4 \\
\hline
\end{tabular}
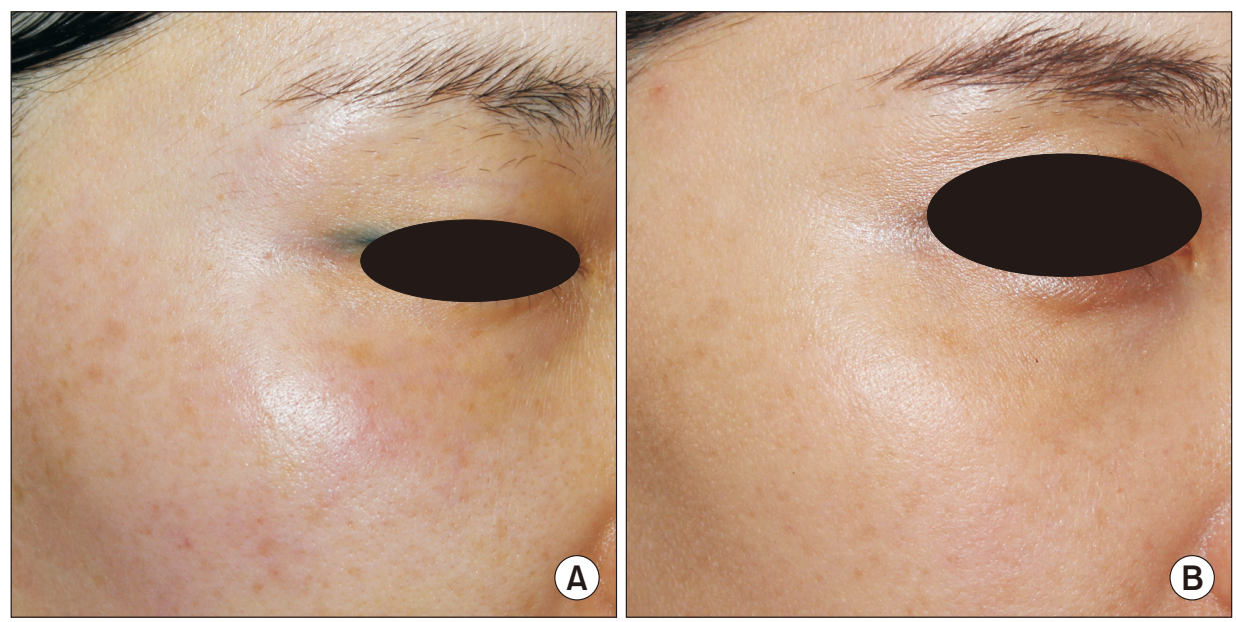

Fig. 2. Photographs of a 33-year-old woman. (A) Preoperative image. (B) Photograph after all three treatment sessions.
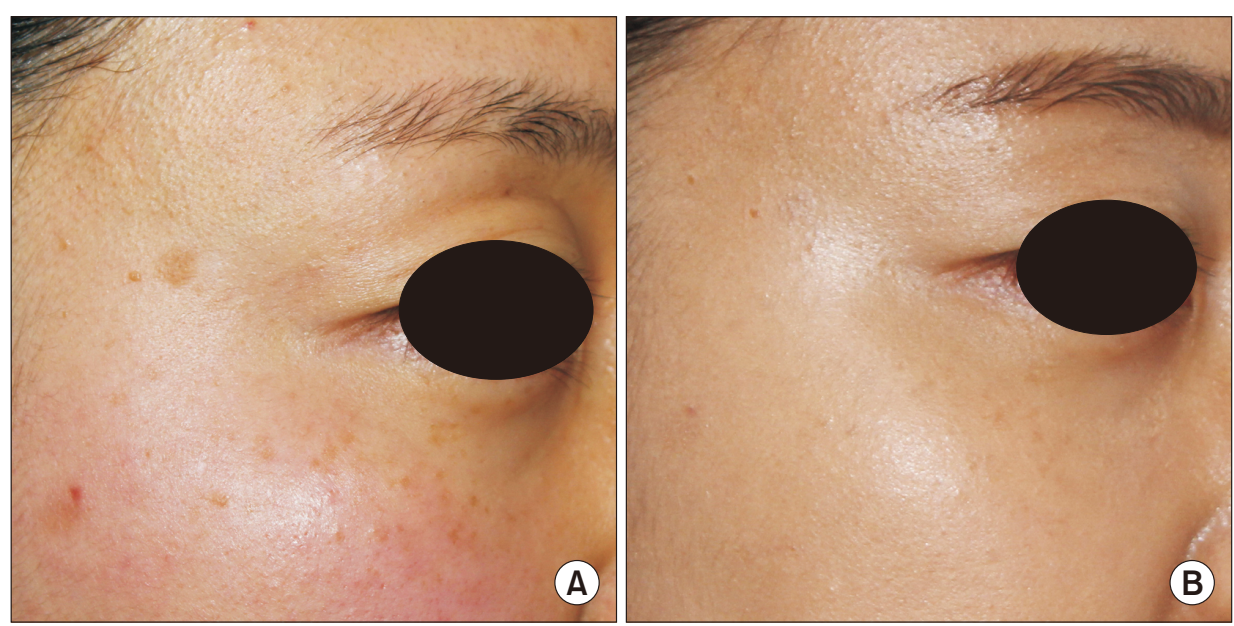

Fig. 3. Photographs of a 37-year-old woman. (A) Preoperative photograph. (B) Photograph after three treatment sessions. 
at 2 weeks after the first procedure, 2 weeks after the second procedure, 2 weeks after the third procedure, and 6 weeks after the third procedure. No serious adverse effects were observed during the 6-month follow-up period (Table 1, Fig. 2, 3).

\section{DISCUSSION}

HIFU burns tissue using high heat $\left(65-100^{\circ} \mathrm{C}\right)$ at the focus where high-intensity ultrasound emergency is collected in one place. If you focus ultrasound energy at about 100,000 times stronger than the intensity of the ultrasonic wave used for diagnosis, heat is generated at the focus area. This is similar to a convex lens, which collects sunlight and generates heat at the focus area. The ultrasound energy itself is harmless to the human body and generates heat only at the focus where the ultrasound energy is concentrated, so plastic surgeons can treat the lesion without the need for general anesthesia or use of a knife or needle. ${ }^{4,5}$

In order to minimize post-treatment adverse events, clinicians have developed various non-invasive skintightening procedures to induce collagen shrinkage and remodeling. Furthermore, ultrasonography is able to penetrate into the subdermal layer and SMAS, and induce thermal coagulation to avoid undesired post-treatment adverse events compared with carbon dioxide laser resurfacing. ${ }^{3}$

Ultrasound energy has characteristics that are suitable for skin lifting and tightening. First, it is believed that ultrasound energy can be transmitted into the deeper subcutaneous layer of the face or even the SMAS, and it is the most effective method for skin lifting and tightening. Second, both the epidermis and dermis can be protected from ultrasound energy during its transmission, reducing the risk of advertent cutaneous layers. ${ }^{5}$

HIFU uses high energy and is mainly used for non-surgical ablation of tumors. HIFU can also be used to ablate adipose tissue for body contouring. Microfocused ultrasonography (MFU) uses much lower energy to treat the superficial layer of the skin and is able to elevate the local temperature higher than $60^{\circ} \mathrm{C}$ to cause collagen contracture. When energy is targeted to discrete areas within the dermal and subdermal tissues, MFU induces discrete thermal coagulation zones while sparing adjacent nontarget tissues. Additionally, the heat induces denaturation and contraction of collagen fibers in the subcutaneous fat layer. ${ }^{6.7}$

There is one more thing to watch out for when performing HIFU on the eye. Ask the patient to look at the opposite side of the procedure site. Have your eyes look down when you are working on your eyes, and when you are working under your eyes. The wrinkles on the surface of the skin of the site are also expanded, which makes it easier to perform the procedure, and even if the skin is deeply mistaken, the probability of reaching the eyeball surface is reduced. ${ }^{8}$

When HIFU is irradiated to the curved area around the eyes, blistering may occur when the skin is not $100 \%$ contacted. Make sure that it is exactly $90^{\circ}$ and that one side of the cartridge does not float with your skin. In this case, an elevated striation may occur.

\section{CONCLUSIONS}

This study suggests that the aging face, with wrinkling and sagging, can be improved by HIFU, while minimizing injury to the epidermis and dermis. In addition, retreatment is recommended at 3 months later to maintain the efficacy of the results.

\section{CONFLICT OF INTEREST}

The authors declare no conflicts of interest.

\section{REFERENCES}

1. Jung NY, Rachmilevitch I, Sibiger O, Amar T, Zadicario E, Chang JW. Factors related to successful energy transmission of focused ultrasound through a skull : a study in human cadavers and its comparison with clinical experiences. J Korean Neurosurg Soc 2019;62:712-22.

2. Aşiran Serdar Z, Aktaş Karabay E, Tatlıparmak A, Aksoy B. Efficacy of high-intensity focused ultrasound in facial and neck rejuvenation. J Cosmet Dermatol. In press 2019.

3. Azuelos A, SidAhmed-Mezi M, La Padula S, Aboud C, Meningaud JP, Hersant B. High-intensity focused ultrasound: a satisfactory noninvasive procedure for neck rejuvenation. Aesthet Surg J 2019;39:NP343-51.

4. Katz B, Bard R, Goldfarb R, Shiloh A, Kenolova D. Ultrasound assessment of subcutaneous abdominal fat thickness after treatments with a high-intensity focused electromagnetic field device: a multicenter study. Dermatol Surg 2019;45:1542-8.

5. Kim SH, Lee SJ, Kim HJ, Lee JH, Jeong HS, Suh IS. Agingrelated changes in the mid-face skin elasticity in East Asian women. Arch Craniofac Surg 2019;20:158-63.

6. Erkiert-Polguj A, Algiert-Zielinska B, Zdunska K, Markiewicz A, Skubalski J, Rotsztejn $\mathrm{H}$. The evaluation of elasticity after nonablative radiofrequency rejuvenation. J Cosmet Dermatol 2019;18:511-6. 
7. Fischer TC, Gauglitz GG. Nonsurgical facial rejuvenation. Facial Plast Surg 2016;32:243-4.

8. Chilukuri S, Lupton J. "Deep heating" noninvasive skin tighten- ing devices: review of effectiveness and patient satisfaction. J Drugs Dermatol 2017;16:1262-6. 